\title{
Efeitos da Liberaçáo Miofascial Sobre a Flexibilidade de um Paciente com Distrofia Miotônica de Steinert
}

\author{
Effects of the Myofascial Release on the Flexibility of a Patient With Myotonic Dystrophy Steinert
}

\author{
Elvyna Melo Rêgo ${ }^{1}$, Marilene Marfin-Martin², Almir Vieira Dibai Filho', \\ Francis Meire Fávero ${ }^{4}$, Acary Souza Bulle Oliveira ${ }^{5}$, Sissy Veloso Fontes ${ }^{6}$
}

\begin{abstract}
RESUMO
Introdução. A distrofia miotônica de Steinert (DMS) é a forma mais frequente das distrofias musculares, de início na idade adulta, merecendo uma atenção fisioterapêutica especializada. Objetivo. Analisar os efeitos da liberação miofascial (LMF) sobre a flexibilidade de um paciente com DMS. Método. Relato de caso de um paciente com DMS, sexo masculino, 37 anos, submetido a 6 sessôes fisioterapêuticas baseadas na técnica de LMF, que consiste em uma abordagem manual da qual decorre uma pressáo interativa entre a mão do terapeuta e as respostas do tecido fascial. Foram realizadas duas avaliaçôes da flexibilidade, uma antes e outra após as sessôes de tratamento. Além disso, o voluntário foi questionado sobre possíveis melhoras em suas atividades diárias após a intervenção com LMF. Resultados. Verificou-se alteraçóes na amplitude de movimento para mais e para menos graus nas articulações avaliadas, em decorrência de uma reorganização estrutural do corpo. Segundo opiniáo do paciente, houve uma melhora da sua condiçâo durante a realizaçáo de suas atividades de vida diária. Conclusáo. A LMF influenciou a função da fáscia e músculo, alterando a flexibilidade articular.
\end{abstract}

Unitermos. Fáscia, Neurologia, Modalidades de Fisioterapia.

Citaçáo. Rêgo EM, Marfin-Martin M, Dibai Filho AV, Fávero FM, Oliveira ASB, Fontes SV. Efeitos da Liberação Miofascial Sobre a Flexibilidade de um Paciente com Distrofia Miotônica de Steinert.

\begin{abstract}
Introduction. The myotonic dystrophy Steinert (MDS) is the most common form of muscular dystrophy, with onset during adulthood, deserving special multiprofessional attention. Objective. Was analyzed the effects of myofascial release (MFR) on the flexibility of a patient with MDS. Method. Case report of a patient with MDS, male, age 37 , submitted to six sessions of physical therapy based on the MFR, which consists of a manual approach by interactive pressure between therapist's hand and fascia. It was performed two assessments of flexibility, one before and one after the treatment sessions. In addition, the volunteer were asked about possible improvements in their daily activities after the intervention with MFR. Results. It was verified changes in range of motion for both increasing and reducing the degrees in joints evaluated, due to structural body reorganization. According to the subjective opinion of patient, there was an improvement in their condition during the course of their daily activities. Conclusion. The MFR influenced the function of fascia and muscle, altering joint flexibility.
\end{abstract}

Keywords. Fascia, Neurology, Physical Therapy Modalities.

Citation. Rêgo EM, Marfin-Martin M, Dibai Filho AV, Fávero FM, Oliveira ASB, Fontes SV. Effects of the Myofascial Release on the Flexibility of a Patient With Myotonic Dystrophy Steinert.

Trabalho realizado no Setor de Investigaçáo de Doenças Neuromusculares, Disciplina de Neurologia Clínica - Departamento de Neurologia e Neurocirurgia, Escola Paulista de Medicina - Universidade Federal de Sáo Paulo (UNIFESP), São Paulo-SP, Brasil.

1.Fisioterapeuta, Mestranda em Fisioterapia, Universidade Metodista de Piracicaba (UNIMEP), Piracicaba-SP, Brasil.

2.Fisioterapeuta, Psicóloga, Mestre em Psicologia da Saúde, Universidade Metodista de São Paulo (UMESP), São Paulo-SP, Brasil.

3.Fisioterapeuta, Mestrando em Fisioterapia, Universidade Metodista de Piracicaba (UNIMEP), Piracicaba-SP, Brasil.

4.Fisioterapeuta, Mestre em Ciências, Universidade Federal de São Paulo (UNIFESP), São Paulo-SP, Brasil.

5.Neurologista, Doutor, Professor do Departamento de Neurologia e Neurocirurgia, Universidade Federal de Sáo Paulo (UNIFESP), São Paulo-SP, Brasil. 6.Fisioterapeuta, Doutora, Universidade Federal de São Paulo (UNIFESP), São Paulo-SP, Brasil.

Endereço para correspondência: Sissy Veloso Fontes Universidade Federal de São Paulo, Departamento de Neurologia e Neurocirurgia. Rua Estado de Israel, 899, Vila Clementino CEP 04022-002, Sáo Paulo-SP, Brasil. E-mail: sissyfontes@gmail.com

Relato de Caso Recebido em: 28/02/11

Aceito em: 12/12/11

Conflito de interesses: não 


\section{INTRODUÇÃO}

Apesar de consideradas raras, as doenças neuromusculares apresentam importância crescente, em decorrência da gravidade, da necessidade de estudos genéticos e biológicos. Mesmo existindo um grande número de doenças, as manifestaçóes clínicas são relativamente pobres, em relação às suas formas de apresentação ${ }^{1}$.

A distrofia miotônica de Steinert (DMS) é a forma mais frequente das distrofias musculares, de início na idade adulta, merecendo uma atenção multiprofissional especial. A prevalência é de aproximadamente 4 casos para cada 100.000 indivíduos. Trata-se de uma doença multissistêmica de herança autossômica dominante ${ }^{2}$. O gene da DMS apresenta uma expressividade muito variável, o que resulta numa grande variabilidade de quadros clínicos entre indivíduos afetados, dentro de uma mesma família e entre famílias diferentes. A idade de instalação e a velocidade de evolução da doença também são muito variáveis ${ }^{3}$.

A DMS é considerada uma doença multissistêmica, pois atinge diferentes órgãos e sistemas causando, além do comprometimento muscular, alteraçôes cardíacas, pulmonares, oculares, ósseas, endócrinas, cutâneas e cognitivas. Ela é caracterizada principalmente por fenômeno miotônico (dificuldade de relaxamento após contração muscular), fraqueza muscular progressiva e atrofia muscular das partes distais dos membros (mãos e pés), do pescoço e da face, levando a disartrofonia e disfagia. Ocorre também fraqueza da musculatura orofaríngea e respiratória, gerando complicações respiratórias. O comprometimento na visão é uma dificuldade marcante causada por uma catarata leve ou mais severa. O retardo mental é comum, podendo assumir uma forma leve ou mais acentuada. Desta forma, o tratamento da DMS deve englobar a minimização dos problemas respiratórios e ortopédicos ${ }^{3}$.

O somatório de alterações comuns aos indivíduos com DMS resulta em consequências diversas, comprometendo a qualidade de vida. Um estudo realizado nesta temática com sujeitos com distrofia miotônica constatou que os mesmos têm uma redução da força muscular expiratória, sendo comuns repercussóes negativas, principalmente, sobre o aspecto físico ${ }^{4}$.

Dentre os diversos procedimentos fisioterapêuticos existentes, a cinesioterapia é considerada a intervenção principal para pacientes com disfunçôes neuromotoras e compreende a terapia que utiliza exercícios e ou atividades físicas. A massoterapia, também utilizada, consiste na mobilização de tecidos moles corporais superficiais e ou profundos 5 . Além disso, ainda fazem parte da mesma as terapias de mobilização de diferentes tecidos e fluidos corporais $^{5}$.

Uma dos recursos fisioterapêuticos que se destaca nos tempos atuais é a liberação miofascial, cujo termo fascia, do latim, significa uma banda ou bandagem e é usado na anatomia macroscópica para designar diferentes estruturas, como todas as estruturas fibrosas de proteção (fáscia peri e intrafaringeana), conjunto orgânico (fáscia endocárdica) e tecidos conjuntivos de nutrição (fáscia superficial e própria), representando assim um conjunto membranoso extenso. Neste contexto, constitui-se numa peça única, que se desdobra sobre si mesma em diferentes espessuras e densidade, de acordo com as exigências funcionais para, ao mesmo tempo, envolver, separar e conectar diferentes estruturas, em diferentes camadas - da fáscia superficial ao endomísio - sobre a qual o menor tensionamento, ativo ou passivo, repercute sobre o conjunto, numa ideia de globalidade ${ }^{6}$.

Portanto, justifica-se esse estudo uma vez que a DMS é uma doença multissistêmica, de caráter progressivo irreversível ${ }^{1,2}$, onde os recursos terapêuticos manuais se destacam como uma das possibilidades fisioterapêuticas para o tratamento desta afecção neurológica. Dessa forma, o estudo de caso em questão teve como objetivo avaliar um destes recursos, a liberação miofascial, sobre a flexibilidade de um paciente com DMS.

\section{MÉTODO}

\section{Relato de caso}

Paciente do sexo masculino, 37 anos, branco, solteiro, analista de sistemas, com DMS (confirmada por avaliação clínica e através de exames subsidiários do nível da CK e DNA), admitido e avaliado no ambulatório de doenças neuromusculares da Universidade Federal de São Paulo. Na história familiar relatou que o pai também apresenta a doença. Realiza natação duas vezes por semana, sendo 60 minutos o tempo de cada treino.

Atualmente apresenta ao exame físico: marcha len- 
tificada, hipertrofia de panturrilhas com predomínio em direita, hipercifose torácica, ptose palpebral, disartrofonia, disfagia moderada. Devido a DMS ser uma doença multissistêmica apresenta ainda cristais de catarata, insuficiência mitral leve, hérnia de hiato, má perfusão periférica, calvície, câimbras e não apresenta miotonia.

A pesquisa foi aprovada pelo Comitê de Ética em Pesquisa da Universidade Federal de São Paulo, sob o protocolo no 2012/09.

\section{Avaliaçáo}

Após entender o protocolo experimental e concordar em participar do estudo, o paciente assinou um termo de consentimento livre e esclarecido.

Foram realizadas duas avaliações, antes e outra após a intervenção fisioterapêutica, no período compreendido entre janeiro e fevereiro de 2010. As avaliaçóes consistiram da mensuração da flexibilidade articular por meio de um flexímetro Code Research Institute ${ }^{\oplus}$, com fixação através de faixas de velcro. Assim, foi avaliada a amplitude de movimento dos ombros, cotovelos, punhos, quadris, joelhos, tornozelos, tronco e cervical.

Após as intervençóes, solicitou-se que o voluntário respondesse ao questionamento: "Quais as mudanças que você percebeu nas suas atividades do dia a dia após as 6 sessóes de Fisioterapia?"

\section{Intervenção fisioterapêutica}

A técnica fisioterapêutica adotada foi a liberação miofascial. A mesma é realizada mediante uma técnica que mescla apoios, pressáo manual e deslizamentos no tecido miofascial, que requer o reconhecimento das áreas e trajetos de resistências e tensóes e que se dá num processo interativo, pois necessita da resposta do corpo do paciente para determinar a duração, profundidade e direção da pressão exercida sobre o tecido. $\mathrm{O}$ toque realizado com as mãos é a sobrecarga inicial colocada sobre o tecido, a partir do qual se esperam as respostas bioquímicas e mecânicas. O que é primeiramente afetado é o componente do complexo elástico colagenoso do tecido (a amplitude elástica) e o terapeuta é capaz de sentir pelo tato uma resistência flexível (o limite elástico).

A intervenção fisioterapêutica foi realizada por uma única fisioterapeuta capacitada e habilitada em apli- car o método, assim como a avaliação também foi realizada por uma única fisioterapeuta. Foram realizadas 6 sessões de liberação miofascial, cada uma com duração de 60 minutos, sendo realizadas uma vez por semana, totalizando um tempo total de tratamento de 1 mês e 15 dias. Assim, os achados alcançados são importantes por retratar os resultados de uma intervenção a curto prazo.

Durante a realização do estudo, o paciente continuou a desempenhar suas atividades laborais, esportivas e recreativas normalmente.

\section{Análise dos dados}

Os resultados estão apresentados em valores absolutos. Utilizou-se a técnica de análise do conteúdo. Assim, conforme descrevem alguns autores, esta se estabelece no texto como meio de expressão do sujeito, em que o analista realiza uma categorização, inferindo uma expressão que o represente ${ }^{6}$.

\section{RESULTADOS}

As Tabelas 1 e 2 apresentam os dados referentes à amplitude de movimento das articulaçóes avaliadas antes e após as sessões de intervenção fisioterapêutica. A flexibilidade dos membros superiores e inferiores apresentou ganhos bilaterais nos seguintes movimentos: extensão e abdução de ombro; flexão, extensão, pronaçãa, desvio ulnar e radial de punho; extensão, rotação medial e abdução de quadril; e flexão dorsal de tornozelo.

Com relação ao tronco, verificou-se uma diminuição da flexibilidade em todos os movimentos avaliados, exceto para rotação lateral à direita, cuja alteração foi nula, conforme mostra a Tabela 3.

A Tabela 4 apresenta a opinião do voluntário acerca das mudanças no seu cotidiano após a intervenção fisioterapêutica.

\section{DISCUSSÃO}

A DMS é considerada uma doença multissistêmica, pois atinge diferentes órgãos e sistemas, causando alteraçôes cardíacas, pulmonares, oculares, ósseas, endócrinas, cutâneas e cognitivas ${ }^{3}$. Ela é caracterizada principalmente por fenômeno miotônico, entendido como uma dificuldade de relaxamento do músculo após contração vigorosa, suas primeiras manifestaçôes são no 
Tabela 1

Distribuição da amplitude de movimento das articulaçôes dos membros superiores do sujeito do estudo antes e após as sessôes de liberação miofascial

\begin{tabular}{|c|c|c|c|c|c|c|}
\hline \multirow{2}{*}{$\begin{array}{c}\text { Articulaçáo } \\
\text { Ombro }\end{array}$} & \multicolumn{3}{|c|}{ Flexibilidade (direita) } & \multicolumn{3}{|c|}{ Flexibilidade (esquerda) } \\
\hline & Antes & Após & Diferença & Antes & Após & Diferença \\
\hline Flexão $\left(0\right.$ a $\left.180^{\circ}\right)$ & $103^{\circ}$ & $124^{\circ}$ & $21^{\circ}$ & $148^{\circ}$ & $135^{\circ}$ & $-13^{\circ}$ \\
\hline Extensão $\left(0 \mathrm{a} 60^{\circ}\right)$ & $39^{\circ}$ & $47^{\circ}$ & $8^{\circ}$ & $40^{\circ}$ & $47^{\circ}$ & $7^{\circ}$ \\
\hline Rotação medial $\left(0\right.$ a $\left.70^{\circ}\right)$ & $63^{\circ}$ & $57^{\circ}$ & $-6^{\circ}$ & $51^{\circ}$ & $85^{\circ}$ & $34^{\circ}$ \\
\hline Rotação lateral (0 a 90º & $78^{\circ}$ & $55^{\circ}$ & $-23^{\circ}$ & $60^{\circ}$ & $44^{\circ}$ & $-16^{\circ}$ \\
\hline Abdução (0 a 180) & $84^{\circ}$ & 970 & $13^{\circ}$ & $84^{\circ}$ & $97 \mathrm{o}$ & $13^{\circ}$ \\
\hline Adução $(0$ a 45º & $46^{\circ}$ & $25^{\circ}$ & $-21^{\circ}$ & $33^{\circ}$ & $29^{\circ}$ & $-4^{\circ}$ \\
\hline Cotovelo & Antes & Após & Diferença & Antes & Após & Diferença \\
\hline Flexáo $\left(0\right.$ a $\left.150^{\circ}\right)$ & $102^{\circ}$ & $113^{\circ}$ & $11^{\circ}$ & $115^{\circ}$ & $109^{\circ}$ & $-6^{\circ}$ \\
\hline Punho & Antes & Após & Diferença & Antes & Após & Diferença \\
\hline Flexão $\left(0\right.$ a $\left.80^{\circ}\right)$ & $33^{\circ}$ & $56^{\circ}$ & $23^{\circ}$ & $36^{\circ}$ & $63^{\circ}$ & $27^{\circ}$ \\
\hline Extensão $\left(0\right.$ a $\left.30^{\circ}\right)$ & $45^{\circ}$ & $47^{\circ}$ & $2^{\circ}$ & $42^{\circ}$ & $60^{\circ}$ & $18^{\circ}$ \\
\hline Supinação $\left(0\right.$ a $\left.80^{\circ}\right)$ & $62^{\circ}$ & $36^{\circ}$ & $-26^{\circ}$ & $46^{\circ}$ & $37 \mathrm{o}$ & -90 \\
\hline Pronação $\left(0\right.$ a $\left.80^{\circ}\right)$ & $56^{\circ}$ & $64^{\circ}$ & $8^{\circ}$ & $55^{\circ}$ & $80^{\circ}$ & $25^{\circ}$ \\
\hline Desvio ulnar $\left(0\right.$ a $\left.30^{\circ}\right)$ & $8^{\circ}$ & $17^{\circ}$ & $9^{\circ}$ & $6^{\circ}$ & $24^{\circ}$ & $18^{\circ}$ \\
\hline Desvio radial $\left(0\right.$ a $\left.20^{\circ}\right)$ & $9^{\circ}$ & $22^{\circ}$ & $13^{\circ}$ & $6^{\circ}$ & $15^{\circ}$ & $9^{\circ}$ \\
\hline
\end{tabular}

Tabela 2

Distribuição da amplitude de movimento das articulaçōes dos membros inferiores do sujeito do estudo antes e após as sessôes de liberação miofascial

\begin{tabular}{|c|c|c|c|c|c|c|}
\hline \multirow{2}{*}{$\begin{array}{c}\text { Articulaçáo } \\
\text { Quadril }\end{array}$} & \multicolumn{3}{|c|}{ Flexibilidade (direita) } & \multicolumn{3}{|c|}{ Flexibilidade (esquerda) } \\
\hline & Antes & Após & Diferença & Antes & Após & Diferença \\
\hline Flexão $\left(0\right.$ a $\left.120^{\circ}\right)$ & $95^{\circ}$ & $87^{\circ}$ & $-8^{\circ}$ & $148^{\circ}$ & $135^{\circ}$ & $-13^{\circ}$ \\
\hline Extensão $\left(0\right.$ a $\left.70^{\circ}\right)$ & $15^{\circ}$ & $20^{\circ}$ & $5^{\circ}$ & $16^{\circ}$ & $18^{\circ}$ & $2^{\circ}$ \\
\hline Rotação medial $(0$ a 45) & $19^{\circ}$ & $33^{\circ}$ & $14^{\circ}$ & $21^{\circ}$ & $35^{\circ}$ & $14^{\circ}$ \\
\hline Rotação lateral ( 0 a 45º) & $35^{\circ}$ & $35^{\circ}$ & $0^{\circ}$ & $34^{\circ}$ & $45^{\circ}$ & $-11^{\circ}$ \\
\hline Abdução $\left(0\right.$ a $\left.45^{\circ}\right)$ & $25^{\circ}$ & $36^{\circ}$ & $11^{\circ}$ & $30^{\circ}$ & $39^{\circ}$ & $9^{\circ}$ \\
\hline Adução $\left(0\right.$ a $\left.30^{\circ}\right)$ & $23^{\circ}$ & $15^{\circ}$ & $-8^{\circ}$ & $12^{\circ}$ & $15^{\circ}$ & $3^{\circ}$ \\
\hline Joelho & Antes & Após & Diferença & Antes & Após & Diferença \\
\hline Flexão $\left(0\right.$ a $\left.135^{\circ}\right)$ & $98^{\circ}$ & $90^{\circ}$ & $-8^{\circ}$ & $119^{\circ}$ & $114^{\circ}$ & $-5^{\circ}$ \\
\hline Tornozelo & Antes & Após & Diferença & Antes & Após & Diferença \\
\hline Flexão dorsal $\left(0\right.$ a $\left.20^{\circ}\right)$ & $11^{\circ}$ & $20^{\circ}$ & $9^{\circ}$ & $10^{\circ}$ & $20^{\circ}$ & $10^{\circ}$ \\
\hline Flexão plantar $(0$ a 50º & $21^{\circ}$ & $20^{\circ}$ & $-1^{o}$ & $23^{\circ}$ & $15^{\circ}$ & $8^{\circ}$ \\
\hline Inversāo ( 0 a 35º) & $18^{\circ}$ & $20^{\circ}$ & $2^{\circ}$ & $16^{\circ}$ & $15^{\circ}$ & $-1^{\circ}$ \\
\hline Eversão $\left(0\right.$ a $\left.15^{\circ}\right)$ & $19^{\circ}$ & $16^{\circ}$ & $-3^{\circ}$ & $18^{\circ}$ & $14^{\circ}$ & $-4^{o}$ \\
\hline
\end{tabular}

músculo esquelético, sendo a característica clínica mais relevante uma debilidade muscular progressiva e atrofia muscular ${ }^{8}$. Neste estudo, de forma excepcional, o voluntário não apresentava miotonia. Logo, observa-se que o diagnóstico deve ser pautado no quadro clínico do paciente e apoiado por exames complementares.
$\mathrm{Na}$ falta de força em alguns músculos e outros não, o indivíduo tende a compensar isto adotando a postura que melhor se ajuste à força da gravidade em todos os movimentos que realiza, gastando dessa forma menos energia. As compensaçóes tendem a ser assimétricas e, por isso mesmo, são geradoras de desconforto, onde o 
Tabela 3

Distribuição da amplitude de movimento do tronco e da cervical do sujeito do estudo antes e após as sessóes de liberação miofascial

\begin{tabular}{cccc}
\hline Articulação & \multicolumn{3}{c}{ Flexibilidade } \\
\hline Tronco & Antes & Após & Diferença \\
\hline Flexão $\left(0\right.$ a $\left.95^{\circ}\right)$ & $41^{\circ}$ & $36^{\circ}$ & $-5^{\circ}$ \\
Extensão $\left(0\right.$ a $\left.35^{\circ}\right)$ & $37^{\circ}$ & $22^{\circ}$ & $-15^{\circ}$ \\
\hline Cervical & Antes & Após & Diferença \\
\hline Flexão $\left(0\right.$ a $\left.65^{\circ}\right)$ & $67^{\circ}$ & $50^{\circ}$ & $-17^{\circ}$ \\
Extensão $\left(0\right.$ a $\left.50^{\circ}\right)$ & $68^{\circ}$ & $53^{\circ}$ & $-15^{\circ}$ \\
Flexão lateral à direita $\left(0\right.$ a $\left.40^{\circ}\right)$ & $56^{\circ}$ & $36^{\circ}$ & $-20^{\circ}$ \\
Flexão latera à esqueda $\left(0 \mathrm{a} 40^{\circ}\right)$ & $49^{\circ}$ & $26^{\circ}$ & $-23^{\circ}$ \\
Rotação lateral à direita $\left(0 \mathrm{a} 40^{\circ}\right)$ & $65^{\circ}$ & $65^{\circ}$ & $0^{\circ}$ \\
Rotação lateral à esquerda $\left(0 \mathrm{a} 40^{\circ}\right)$ & $75^{\circ}$ & $70^{\circ}$ & $-5^{\circ}$ \\
\hline
\end{tabular}

Tabela 4

Resposta do voluntário da pesquisa à pergunta "Quais as mudanças que você percebeu nas suas atividades do dia a dia após as 6 sessöes de Fisioterapia?"

\begin{tabular}{|c|l|}
\hline Técnica de análise & \multicolumn{1}{|c|}{ Resposta do voluntário } \\
\hline \multirow{3}{*}{ Análise do conteúdo } & $\begin{array}{l}\text { Eu senti uma melhora no meu desempenho } \\
\text { para atividades como caminhada e treino } \\
\text { de nataçáo. Aumentou o tempo de duração } \\
\text { da minha caminhada com menos cansaço } \\
\text { e aumentou o tempo do nado imerso com } \\
\text { menor intervalo de descanso. Percebo uma } \\
\text { melhora na minha resistência ao exercício } \\
\text { físico. }\end{array}$ \\
\hline
\end{tabular}

corpo continua sob ação da gravidade ao mesmo tempo em que perde de forma progressiva o alinhamento vertical de sua organização tridimensional. Na tentativa de evitar o desconforto, novas compensaçóes vão se desenvolvendo, afetando progressivamente a funcionalidade de todo o organismo, podendo causar retraçóes não aparentes ao nível de fáscia e músculo. Isso se explica pela reciprocidade entre os sistemas, que se mantêm conectados pelo tecido conjuntivo, sendo possíveis implicações patológicas na saúde como um todo do indivíduo?.

Ao realizar a LMF, regiōes do corpo são liberadas de retraçôes, aumentando a flexibilidade, e, em contrapartida, outras regióes se retraem em prol de uma reorganização estrutural e biomecânica favorável à postura e à realização das atividades funcionais. Este novo padrão estrutural favorável é corroborado pela análise qualitativa da resposta do voluntário.
A relação entre as estruturas ocorre através da fáscia, que separa e ao mesmo tempo une cada uma, dando suporte físico ao adentrar no interior das células pela conexão com o citoesqueleto. Além disso, a matriz extracelular, na qual a fáscia está embebida, é um ambiente único externo com o qual cada célula interage com o seu metabolismo e, nesse sentido, a fáscia novamente se destaca, dessa vez, como estrutura de suporte funcional às demais ${ }^{9}$. Por isso a eleição da liberação miofascial como técnica a ser aplicada no sujeito do estudo, já que se relaciona com a flexibilidade e a elasticidade corporal, tendo, portanto, grande capacidade de alterar funçôes de uma forma holística ${ }^{10}$.

Experimentos já evidenciaram que os tecidos tendinosos e septos intermusculares exercem importante papel no controle de movimentos isocinéticos das articulaçóes, pela força e capacidade de armazenamento de energia durante a contração, alongando mais do que os músculos em várias atividades, dentre elas a deambulação, corrida, e vários esportes que impliquem em força de impulsão, como boxe, arremessos, arco e flecha, dentre outros $^{11}$.

Além da participação fundamental da fáscia na transmissão de forças no sistema musculoesquelético, constatou-se, mediante análise do conteúdo da resposta do voluntário da pesquisa, um aumento na resistência ao exercício e diminuição da fadiga. As técnicas de terapia manual têm sido descritas pela melhora da mobilidade da caixa torácica, costelas, melhora na função pulmonar e qualidade de vida, aumento da oxigenação arterial e retorno linfático ${ }^{12}$, proporcionando uma melhor capacidade em realizar exercícios dinâmicos com grandes grupos musculares, como caminhar, nadar e pedalar, por períodos de tempo prolongados ${ }^{13}$. Novamente, salienta-se a ação global da fáscia sobre o organismo ${ }^{14,15}$.

A melhora da locomoção do paciente está relacionada à capacidade da LMF de agir sobre o tecido conjuntivo, afetando sua viscoelasticidade e, ao mesmo tempo, a função estática ou tônica da fisiologia da locomoção repercutindo, dessa forma, no tensionamento ativo ou passivo sobre todo o conjunto de estruturas ${ }^{16}$.

Portanto, podemos afirmar que: a) houve uma remodelagem da fáscia no organismo do voluntário do estudo; b) que isso ocorreu através de um melhor equilíbrio 
de peso e de forças ao longo do seu eixo vertical; c) que isso contribui favoravelmente para o conjunto dos movimentos que ele realiza, pois a reorganização estrutural que seu corpo revelou foi de melhor postura ortostática e a melhora funcional foi de redução de esforço e melhor integração dos movimentos ao realizar suas atividades de vida diária e esportiva.

\section{CONCLUSÃO}

Os resultados encontrados evidenciam que a terapêutica utilizada, liberação miofascial, influenciou a flexibilidade articular, com capacidade de alterar a amplitude de movimento para mais e para menos graus, em diferentes articulações no sentido de uma aproximação em relação à normalidade, proporcionando ao indivíduo uma reorganizaçáo estrutural e funcional. Soma-se a isto a opinião subjetiva do paciente de melhora da sua condição durante a realização das suas atividades do cotidiano, incluindo esporte, após as sessões fisioterapêuticas.

Sugere-se a realização de estudos futuros com o objetivo de verificar os efeitos da liberação miofascial a longo prazo, uma vez que o estudo em questão se deteve a um período de pouco mais de um mês, o que correspondeu a seis sessões de tratamento.

\section{REFERÊNCIAS}

1.Oliveira ASB, Levy JA. Afecçôes Neurológicas Periféricas. In: Calia LC, Annes M. Reabilitação em Doenças Neuromusculares: Guia Terapêutico Prático. São Paulo: Atheneu Editora; 2003, p.31-64.

2.Harper PS. Myotonic disorders. In: Engel AG, Banker BQ. Myology. New York: McGraw-Hill; 1986, p.1267-96.

3.Otsuka MA, Boffa CFB, Vieira ABAM. Distrofias Musculares: Fisioterapia Aplicada. Rio de Janeiro: Editora Revinter; 2005, p.2-28.

4.Araújo TL, Resqueti VR, Bruno S, Azevedo IG, Dourado Júnior ME, Fregonezif G. Respiratory Muscle Strength and Quality of Life in Myotonic Dystrophy Patients. Rev Port Pneumol 2010;16:892-8.

5.Fontes SV, Alves MAF, Ottoboni C, Fukujima MM. Classificação dos Procedimentos Fisioterápicos. In: Fontes SV, Fukujima MM, Cardeal JO. Fisioterapia Funcional: Fundamentos Para a Prática. São Paulo: Editora Atheneu; 2007, p.149-259.

6.Levy JA. Classificação das Miopatias. In: Levy JA. Doenças Musculares: Estudo Clínico e Diagnóstico. São Paulo: Editora Atheneu; 2001, p.19-33.

7.Caregnato RCA, Mutti R. Pesquisa Qualitativa: Análise de Discurso Versus Análise de Conteúdo. Texto Contexto Enferm 2006;15:679-84.

http://dx.doi.org/10.1590/S0104-07072006000400017

8.Lins LL, Padovani CMCA, Lucena R, Argolo LS, Fernandes LC, Guimarães CVNF, et al. Achados Audiológicos em Indivíduos com Distrofia Miotônica de Steinert. Rev Soc Bras Fonoaudiol 2008;13:344-51.

9.Martin MM. Efeito da Liberação Miofascial em Esclerose Sistêmica Difusa: Relato de Caso. Rev Rolfing Brasil 2009;9:3-9.

10.Leite JAM, Matutino RRB, Aragão JHD. Efeito da Liberação Miofascial dos Isquiotibiais na Amplitude do Movimento do Quadril. Ter Man 2008;6:154-8.

11.Chino K, Oda T, Kurihara T, Nagayoshi T, Yoshikawa K, Kanehisa H, et al. In Vivo Fascicle Behavior of Synergistic Muscles in Concentric and Eccentric Plantar Flexions in Humans. J Electromyogr Kinesiol 2008;18:79-88.

http://dx.doi.org/10.1016/j.jelekin.2006.08.009

12.Campelo GO. A Manipulaçấo do Tecido Conjuntivo no Tratamento da Dor Lombar Crônica de Origem Miofascial. Ter Man 2008;6:307-13.

13. Kisner C, Colby LA. Exercício Fisioterapêutico: Fundamentação Conceitual. In: Kisner C, Colby LA. Exercícios Terapêuticos: Fundamentos e Técnicas. $4^{a}$ ed. São Paulo: Editora Manole; 2005, p. 3-33.

14.Findley T. Fascia Research II: Second International Fascia Research Congress. International Journal of Therapeutic Massage and BodyWork 2009;2:4-9. 15.Bianchini LP, Moreira MR. Influência da Manipulação Miofascial Sobre a Amplitude Articular. Ter Man 2003;2:78-80.

16. Campelo GO. A Manipulaçáo do Tecido Conjuntivo no Tratamento da Dor Lombar Crônica de Origem Miofascial. Ter Man 2008;6:307-13. 